\title{
Multiplex recurrence networks
}

\author{
Deniz Eroglu, ${ }^{1,2,{ }^{*}}$ Norbert Marwan, ${ }^{1}$ Martina Stebich, ${ }^{3}$ and Jürgen Kurths ${ }^{1,2,4}$ \\ ${ }^{1}$ Potsdam Institute for Climate Impact Research (PIK), Potsdam 14473, Germany \\ ${ }^{2}$ Department of Physics, Humboldt University, 12489 Berlin, Germany \\ ${ }^{3}$ Senckenberg Research Station of Quaternary Palaeontology Weimar, Am Jakobskirchhof 4, Weimar 99423, Germany \\ ${ }^{4}$ Institute for Complex Systems and Mathematical Biology, University of Aberdeen, Aberdeen AB24 3UE, United Kingdom
}

(Received 13 July 2017; published 25 January 2018)

\begin{abstract}
We have introduced a multiplex recurrence network approach by combining recurrence networks with the multiplex network approach in order to investigate multivariate time series. The potential use of this approach is demonstrated on coupled map lattices and a typical example from palaeobotany research. In both examples, topological changes in the multiplex recurrence networks allow for the detection of regime changes in their dynamics. The method goes beyond classical interpretation of pollen records by considering the vegetation as a whole and using the intrinsic similarity in the dynamics of the different regional vegetation elements. We find that the different vegetation types behave more similarly when one environmental factor acts as the dominant driving force.
\end{abstract}

DOI: 10.1103/PhysRevE.97.012312

\section{INTRODUCTION}

In order to understand the dynamical behavior of systems in a broad range of scientific fields such as physics, biology, medicine, climatology, economy, etc., time series analysis provides crucial techniques. Although investigation of time series can be done by various techniques, such as basic statistics, symbolization, power spectra, or similarity analysis, phase space based methods have become an important role in dynamical systems' analysis. Recurrence of a trajectory in its phase space is one of the most important fundamental features of dynamical systems. Related approaches have been used for several decades. The first recurrence based approach is known as the Poincaré recurrence theorem introduced in 1890 [1]. The theorem indicates that almost all trajectories of dynamical systems will turn back infinitesimally close to their previous positions after a sufficiently long but finite time [1]. Continuous dynamical systems can be defined by a set of ordinary differential equations. These equation sets are called flows and if the phase space of a flow has a bounded volume, then the Poincaré recurrence theorem is always valid [2]. Among the many different approaches of analyzing dynamical systems by their recurrence, the recurrence plot (RP) is a multifaceted and powerful approach to study different aspects of dynamical systems. Introduced by Eckmann et al. in 1987, the RP is a matrix to show the times of recurrences of a trajectory in its phase space [3]. Afterwards, many statistical quantification methods based on RP were developed for characterizing dynamical properties, regime transitions, synchronization, etc. [4].

Understanding underlying dynamics and detecting possible regime changes in the evolution of dynamical systems are important problems studied by time series analysis. For instance,

*eroglu@pik-potsdam.de we assume a dynamical system

$$
\overrightarrow{\dot{x}}=f(\vec{x}, r),
$$

where $\vec{x} \in \mathbb{R}^{m}, f: \mathbb{R}^{m} \rightarrow \mathbb{R}^{m}$, and a control parameter $(r)$ that has not to be constant on time $r=g(t)$. The purpose of the analysis is to detect possible dynamical regime changes in the time series caused by the time dependence of $r$. Transitions in the dynamics can be detected by different RP based measures, which in general are powerful to study complex, real-world systems [5-7]. Examples of their successful application to realworld systems have been found in medicine [6,8-12], Earth science [7,13-17], astrophysics [18,19], electrochemistry [20], and others [21-23].

In the last decade, transformation of a time series to a complex network has become a very powerful approach to analyze complex dynamical systems. There are several ways to convert a time series to a network such as symbolic dynamics based techniques [24,25], visibility graphs [26-28], cycle networks [29], or recurrence networks (RNs) [30-32]. In this work we consider recurrence based approaches, since it is well known that recurrences are a thumbprint of characteristic properties of dynamical systems [22,33-37]. Moreover, recently we have presented a work on fundamental behavior of recurrence plot measures which proves that the Huberman-Rudnick unique scaling is valid for the RP measures, while changing the control parameter of a given dynamical system [38]. The adjacency matrix of a complex network represents the structure of the system and thus determines the links between the nodes of a network. For unweighted and undirected networks, the adjacency matrix is binary and symmetric, hence very similar to an RP. As a consequence, we know that there is a similar unique scaling behavior for RN measures and RNs have been used to investigate real-world systems such as the climate [7] or the cardiorespiratory system [39].

Naturally many real systems possess many degrees of freedom and such systems can be described by multivariate time 
series. Each component of these systems can be considered as a time series and we can reconstruct a phase space with them. Meanwhile, when the number of components is huge, we need to have longer time series for enough occurrence of recurrences in the phase space in order to analyze the system by the recurrences. However, in several disciplines like astrophysics, Earth sciences, and economy, having long time series cannot be ensured. Therefore the components are analyzed one by one or some dimension reduction is applied, but these might result in further information loss from the system.

An increasing number of dimensions requires longer time series for traditional recurrence networks. Usually, the period of a trajectory in the phase space is extending with an increasing number of dimensions. With other words, if the dimension of the phase space is increasing, we need longer and longer time series to have enough number of recurrences to analyze the given system. Therefore, traditional RNs are not efficiently applicable for short multivariate time series to interpret the behavior of the system, because of the scarcity of recurrences. In order to gain the maximum yield from data sets which has many degrees of freedom, in this work we introduce a multiplex network-based approach to analyze multivariate time series. Network modeling is a main tool in characterizing a broad range of problems in physics, biology, social interactions, Earth science, economy, etc. If there are interactions between components of the system they can be modeled by a coupled network structure. For example, the RN approach has been used to identify the direction of intersystems relationships between bivariate time series [40]. Now we represent each component of the system as a separated $\mathrm{RN}$ and interpret it as a layer of a multiplex network [41-45] that we call a multiplex recurrence network (MRN). A similar approach based on visibility graphs was recently discussed by Lacasa et al. [28], resulting in multiplex visibility graphs [28]. Using the measures given by Lacasa et al. [28], we will show a comprehensive comparison of regular RNs and multiplex recurrence networks on coupled chaotic systems. By employing the recurrence properties of the dynamical system for the multiplex network construction, we focus on the dynamics, whereas the visibility graph is more restricted to the statistical properties of the system (e.g., the Hurst exponent) [27,32]. We demonstrate our method's efficiency by investigating high dimensional systems which are not possible to analyze by the traditional $\mathrm{RN}$ approach directly.

As a real-world application, we analyze a palaeoclimate record which is a multivariate data set of pollen taxa representing the variability of past vegetation in NE China over the Holocene and found significant variations in the congruence of the vegetation dynamics of the considered tree species.

\section{METHODS}

Recurrence based techniques have been successfully used for time series analysis of physical, biological, economical, climate systems. Multilayer networks have been recently introduced as a powerful representation of a specific network of networks. In this section, we discuss RNs and how to use them in multilayer networks in order to analyze multivariate time series.

\section{A. Recurrence networks}

A time series $\left\{u_{i}\right\}_{i=1}^{N}$ can be reconstructed as a trajectory [Eq. (1)] in its phase space with time delay embedding [46],

$$
\mathbf{x}_{i}=\left(u_{i}, u_{i+\tau}, \ldots, u_{i+\tau(M-1)}\right),
$$

where $M$ is the embedding dimension which can be found by a false nearest-neighbors approach and $\tau$ is the embedding delay which can be computed by mutual information or autocorrelation [47].

Two state vectors of the reconstructed time series are considered to be recurrent if the second vector falls into the neighborhood (an $\epsilon$-radius sphere) of the first vector. For the trajectory $\vec{x}_{i}\left(i=1, \ldots, N, \vec{x}_{i} \in \mathbb{R}^{M}\right)$, the adjacency matrix of $\mathrm{RN}, \mathbf{A}$, is defined as

$$
A_{i, j}(\epsilon)=\Theta\left(\epsilon-\left\|\vec{x}_{i}-\vec{x}_{j}\right\|\right)-\delta_{i, j}, i, j=1, \ldots, N,
$$

where $N$ is the trajectory length, $\Theta(\cdot)$ is the Heaviside function, $\|\cdot\|$ is the Euclidian norm, and $\delta_{i, j}$ is the Kronecker delta $\left(\delta_{i, j}=1\right.$ if $i=j$, otherwise $\left.\delta_{i, j}=0\right)$ [31,48].

A RN is constructed in the following way: Consider the time points of a time series as nodes of a network; if the nodes are sufficiently close to each other, in other words, if the space vectors are neighbors, then a link between them is drawn. A represents the network, where $A_{i, j}=1$ if $i$ and $j$ are connected, otherwise $A_{i, j}=0$.

In all recurrence based applications, the threshold $\epsilon$ is an arbitrarily selected small number. The selection of $\epsilon$ can affect the results easily. In order to have a reasonable analysis, some threshold selection techniques were proposed $[4,48,49]$. However none of them is a certain way to choose the threshold. To be consistent, in this work we always used the way which depends on the standard deviation of the time series [50].

\section{B. Multiplex and weighted recurrence networks}

Multiplex structure. In this work, an $m$-layer multiplex network is constructed by RNs. For $m$-dimensional multivariate time series, we can create $m$ different RNs which have the same number of nodes and each node is labeled by its associated time. These networks will form the different layers of a multilayer network. The layers are connected each other with the same time labeled nodes. This procedure requires that the time sampling is the same for all of the used time series. If a multilayer network consists of $m$ layers which has the same number of nodes and the connections between layers are only between a node and its counterpart in the other layers, then we call such networks "multiplex." Networks, transformed from multivariate time series, are compatible with the definition of multiplex networks, because each node is uniquely assigned to a certain time point of the multivariate time series, i.e., we will find the equally time-labeled nodes in all layers.

We consider an $m$-dimensional multivariate time series $\{\boldsymbol{u}(t)\}_{t=1}^{N}$, with $\boldsymbol{u}(t)=\left(u_{1}(t), u_{2}(t), \ldots, u_{m}(t)\right) \in \mathbb{R}^{m}$ for any value of $t$. Then, the RN of the $\kappa$ th component of $\boldsymbol{u}(t)$ is created and located into the associated layer $\kappa$ of the multiplex network. For an example with $N=3$ time series these steps are illustrated in Fig. 1. First, we have three time series and construct a phase space for each signal. The RN of the $\kappa$ th component of the time series is calculated by Eqs. (2) and (3) and then placed into layer $\kappa$. We denote the adjacency matrix 


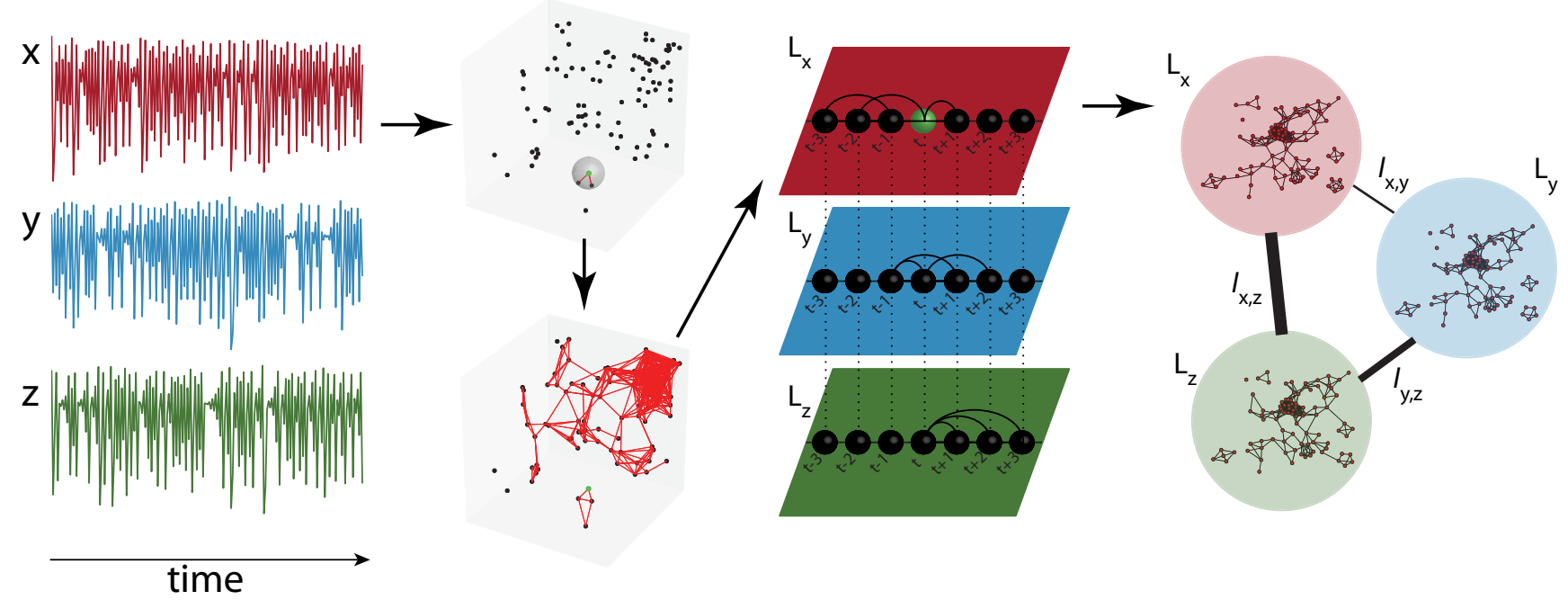

FIG. 1. Illustration of the procedure for generating network structures from a multivariate time series: multivariate time series $\rightarrow$ recurrence networks $\rightarrow$ multiplex recurrence network $\rightarrow$ weighted network of recurrence networks. Dashed lines between multiplex networks' layers connect all layers to all.

of the $\kappa$ th layer as $A^{[\kappa]}=a_{i j}^{[\kappa]}$ and $a_{i j}^{\kappa}=1$ if nodes $i$ and $j$ are connected in layer $\kappa, a_{i j}^{\kappa}=0$ otherwise. The giant adjacency matrix describing the entire multiplex network is denoted by

$$
\mathcal{A}=\left[\begin{array}{cccc}
\mathbf{A}^{[1]} & \boldsymbol{I}_{N} & \cdots & \mathbf{I}_{N} \\
\mathbf{I}_{N} & \mathbf{A}^{[2]} & \ddots & \vdots \\
\vdots & \ddots & \ddots & \mathbf{I}_{N} \\
\mathbf{I}_{N} & \ldots & \mathbf{I}_{N} & \mathbf{A}^{[m]}
\end{array}\right],
$$

where $\mathbf{I}_{N}$ is the identity matrix of size $N$.

In order to measure the similarity between layer $\kappa$ and $\gamma$ of the MRN, we use the interlayer mutual information $I_{\kappa, \gamma}[28]$ :

$$
I_{\kappa, \gamma}=\sum_{k^{[\kappa]}} \sum_{k^{[\gamma]}} P\left(k^{[\kappa]}, k^{[\gamma]}\right) \ln \frac{P\left(k^{[\kappa]}, k^{[\gamma]}\right)}{P\left(k^{[\kappa]}\right) P\left(k^{[\gamma]}\right)},
$$

where $P\left(k^{[\kappa]}\right)$ and $P\left(k^{[\gamma]}\right)$ are the degree distributions of RNs at layer $\kappa$ and $\gamma$ respectively, and $P\left(k^{[\kappa]}, k^{[\gamma]}\right)$ is the joint probability of the existence of nodes which has $k^{[\kappa]}$ degree at layer $\kappa$ and $k^{[\gamma]}$ at layer $\gamma$. The degree distribution $P(k)$ is a probability distribution function which holds a general structure information that how many nodes have each degree. The mutual information measures how much a system is similar to another. Instead of computing mutual information between original time series, degree distributions are considered in Eq. (5). The difference of the commonly used direct mutual information of time series is that $I_{\kappa, \gamma}$ does not compare the probability of states (estimated from the time series) but the topological structure in the phase space based on the recurrences (using the recurrence matrix). This similarity measure $I_{\kappa, \gamma}$ quantifies the information flow between the multiplex networks and, thus, the characteristical behavior of the system. The average of the quantity of $I_{\kappa, \gamma}$ over all possible pairs of layers of MRN gives a scalar variable $I=\left\langle I_{\kappa, \gamma}\right\rangle_{\kappa, \gamma}$ which captures the mean similarity of the degree distributions of the RNs, i.e., the order of coherence in the system.

Another measure to quantify the coherence of the original multivariate system by the MRN is the average edge overlap:

$$
\omega=\frac{\sum_{i} \sum_{j>i} \sum_{\kappa} a_{i j}^{[\kappa]}}{m \sum_{i} \sum_{j>i}\left(1-\delta_{0, \sum_{\kappa} a_{i j}^{[\kappa]}}\right)},
$$

where $\delta_{i j}$ is the Kronecker delta symbol. This measure represents the average number of identical edges over all layers of the multiplex network [28]. Like the interlayer mutual information Eq. (5), $\omega$ estimates the similarity and coherence with averaged existence of overlapped links from nodes $i$ to $j$ between all layers $\kappa$ and $\gamma$. Note that $\omega$ can take values in the interval $[1 / m, 1]$, if the link between $i$ and $j$ occurs in only different layers $\omega=1 / m$, i.e., $a_{i j}^{[\kappa]}=1$ and $a_{i j}^{[\gamma]}=0, \forall \gamma \neq \kappa$. If all links are identical in all layers then $\omega=1$.

Weighted structure. Another approach is the projection of all layers onto one weighted network representation. Now we consider each single layer of an MRN as a node and weighted edges between nodes $\kappa$ and $\gamma$ are determined by the quantity $I_{\kappa, \gamma}$, Eq. (5).

In order to quantify high-dimensional systems, converting multilayer systems to weighted structures is computationally a very efficient approach. The adjacency matrix of the multiplex network $\mathcal{A}$ is a giant matrix $(N m \times N m)$, but in the weighted network case the size of the matrix is only $N \times N$. Furthermore, the quantification of weighted networks is a well-developed analysis [51,52]. Among many measures of weighted networks, we use the clustering coefficient $\mathcal{C}_{w}$ and the average path length $\mathcal{L}_{w}$ in order to detect the transitions between different dynamical regimes. The weighted network clustering coefficient is given by

$$
\mathcal{C}_{w}=\frac{1}{m} \sum_{\kappa} \frac{1}{k_{\kappa}\left(k_{\kappa}-1\right)} \sum_{\gamma} \sum_{\beta}\left(I_{\kappa, \gamma} I_{\kappa, \beta} I_{\gamma, \beta}\right)^{1 / 3},
$$


where $k_{\kappa}$ is the degree of node $\kappa$. The average shortest path length of the weighted network is

$$
\mathcal{L}_{w}=\sum_{\kappa, \gamma \in V} \frac{d(\kappa, \gamma)}{m(m-1)},
$$

where $V$ is the set of nodes (layers of multiplex network) in the weighted network and $d(\kappa, \gamma)$ is the weighted shortest path length between nodes $\kappa$ and $\gamma$.

Both MRN measures, $I$ and $\omega$, represent the similarities in the linking structures of the RNs and have higher values for more similar ones. For example, for periodic systems, even if there is phase difference, the corresponding RNs would be rather similar, resulting in high values for $I$ and $\omega$. When the systems are more chaotic and have finally a more different recurrence structure, $I$ and $\omega$ will decrease. This is similar for $\mathcal{C}_{w}$, since in periodic cases the number of triangle structures in networks is increasing. However, it is opposite for $\mathcal{L}_{w}$ because the diameter of a denser network is in general smaller.

\section{COUPLED MAP LATTICES}

As a first application, we consider multicomponent dynamical systems, namely coupled map lattices (CMLs), which are discrete-time models of diffusively coupled oscillators on a ring model of $m$ sites. CMLs are well-studied dynamical systems that model the behavior of nonlinear systems and exhibit a variety of phenomena [53],

$$
x_{t+1}^{[\kappa]}=(1-\varepsilon) f\left(x_{t}^{[\kappa]}\right)+\frac{\varepsilon}{2}\left[f\left(x_{t}^{[\kappa-1]}\right)+f\left(x_{t}^{[\kappa+1]}\right)\right] .
$$

CMLs have been analyzed with various techniques to quantify their very rich dynamics. Now we compare multiplex and regular RN approaches for CMLs of $m=5$ diffusely coupled chaotic logistic maps $f(x)=4 x(1-x)$, which show interesting dynamics in the range of the control parameter $\varepsilon \in[0,0.4]$ studied here with an increment of $\Delta \varepsilon=0.005$. For instance, pattern selections, high-dimensional chaos regimes, and many different forms of partially synchronized chaotic states occur. We compute a time series of length $N=15000$ for each value of $\varepsilon$. In order to discard transients, we delete the first 10000 values, resulting in time series consisting of 5000 values that have been used for all analyses of the CMLs in this paper. All simulations of CMLs are repeated and averaged over 100 realizations, since the system strongly depends on the initial conditions. In order to compare MRN and RN techniques, we construct a five-dimensional phase space for regular RNs and five separated single RNs for each layer of MRN.

For consistency, we use a recurrence threshold that is proportional to the standard deviation $\sigma$ of the data [4]. In this work, we use $\epsilon=0.05 \sigma$. The logistic map is a one-dimensional dynamical system, therefore embedding is not required for applying recurrence networks in the layers of MRN.

Although both techniques could detect the transitions from fully developed turbulence (FDT), $\varepsilon \in[0.0,0.15]$ and $\varepsilon \in[0.19,0.285]$, to periodic pattern selection (PPS), $\varepsilon \in$ $[0.15,0.19]$, the RN approach cannot distinguish the transition from FDT to spatiotemporal intermittency (STI), $\varepsilon \in$ $[0.285,0.4]$ (Figs. 2 and 3). The multiplex network's measures ( $I$ and $\omega$ ) can recognize every single transition and, especially
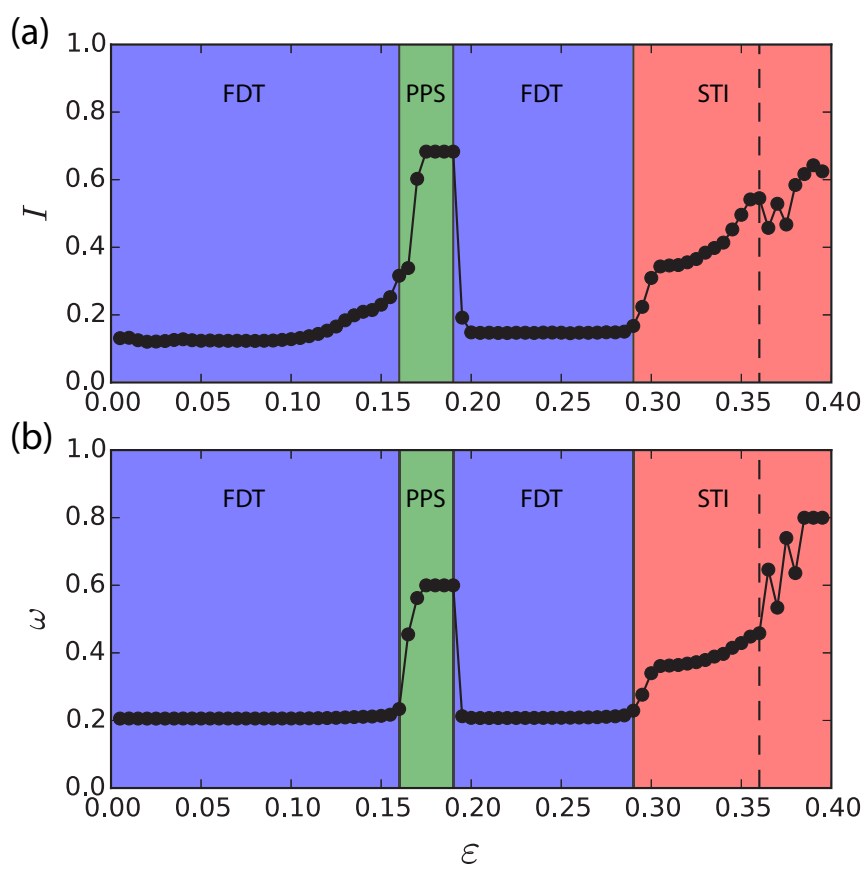

FIG. 2. Results for $m=5$ CMLs [Eq. (9)], (a) average mutual information $(I)$, and (b) average edge overlap $(w)$ of MRN.

$\omega$, can distinguish splitting of trajectories into two attractors in STI at $\varepsilon=0.36$ very clearly (Fig. 2). The MRNs are more sensitive to regime changes than the RNs as well as the MRNs are applicable on large system sizes when RNs are not suitable to deal with them.

Large systems. Simultaneous analysis of interacting components of a system is very important for deep understanding

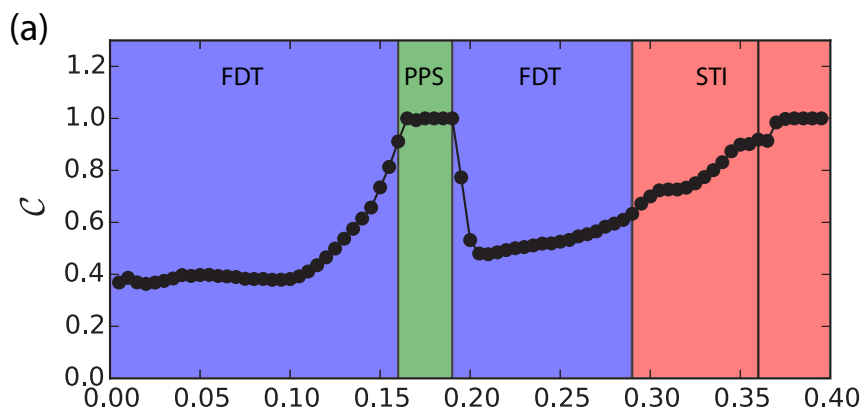

(b)

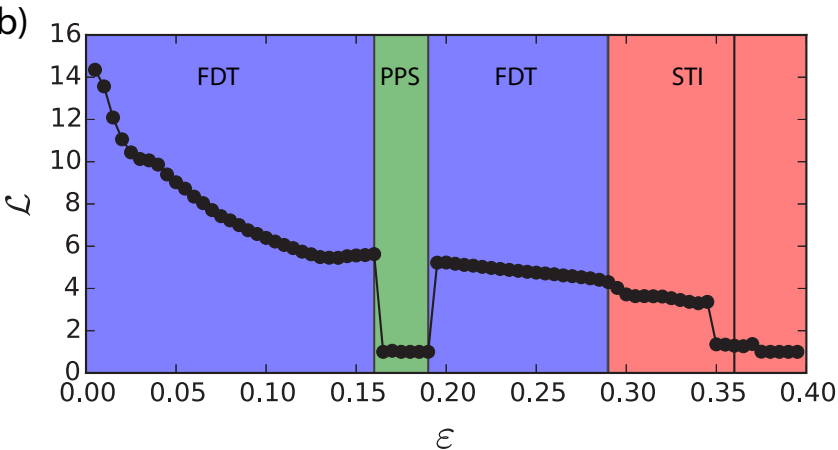

FIG. 3. Results for $m=5$ diffusively coupled map lattices CMLs, (a) clustering coefficient $\mathcal{C}$, and (b) average path length $\mathcal{L}$ of the (regular) recurrence network. 

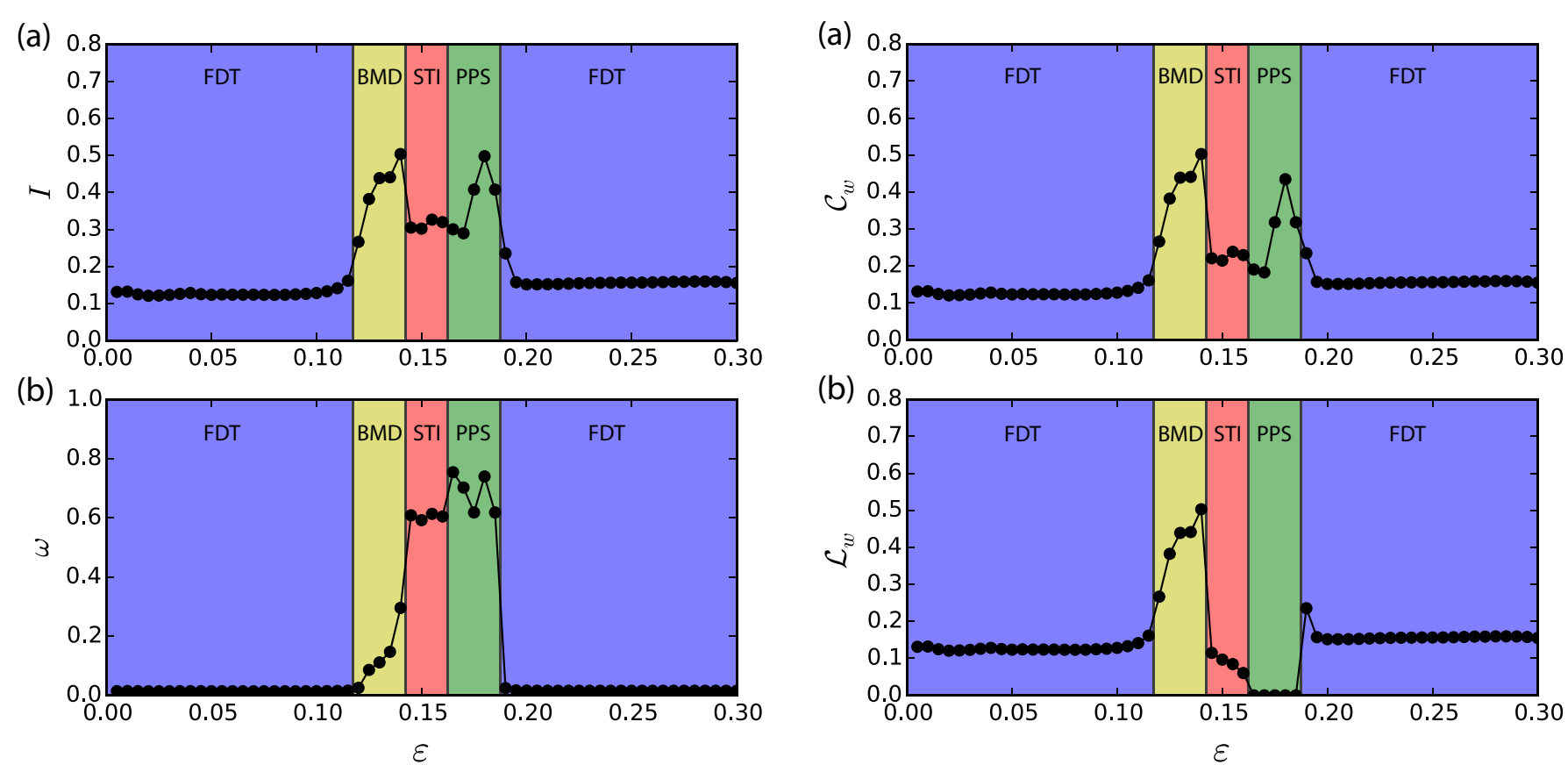

FIG. 4. Results for $m=200$ diffusively coupled map lattices CMLs (a) average mutual information $(I)$, (b) average edge overlap $(w)$ of multiplex network.

of the underlying dynamics. The RN approach is not an appropriate technique to analyze such systems, because while the number of degrees of freedom is increasing, the size (volume) of its phase space is getting larger as well. Therefore, in order to analyze such systems, we need very long data sets proportional to the size of the dimension of the system for establishing recurrences. Although the recurrence based analysis nicely deals with analyzing short time series, finding long data is a common problem in time series analysis. However, MRNs overcome this problem since they use each component of the system as a layer of the giant network.

In this application, we use the same parameters of the RN and the isolated dynamics as in the small system size one. Figure 4 presents the results of the MRN for $m=200$ diffusely coupled logistic maps, which possess one more different regime than the small system, called the Brownian motion of defect (BMD), $\varepsilon \in[0.12,0.14]$. FDT occurs for $\varepsilon \in[0.0,0.12]$ and $\varepsilon \in[0.19,0.30]$, STI is observed for $\varepsilon \in[0.14,0.16]$, and PPS for $\varepsilon \in[0.16,0.19]$. These regimes were observed and presented in detail in [54,55]. The MRN distinguishes the transitions between different regimes clearly and this analysis is a recurrence based approach that detects these transitions in a large coupled system.

Instead of analyzing the giant adjacency matrix of the MRN, we can investigate the same system with taking advantage of the well-developed weighted network analysis. Among the weighted network measures, the clustering coefficient Eq. (7) and the average shortest path length Eq. (8) are computed for the associated network and the results are given in Fig. 5. As a result of MRN, the related weighted network shows the transition very clearly. For large enough multidimensional systems, both approaches can be used.

FIG. 5. Results for $m=200$ diffusively coupled map lattices CMLs (a) clustering coefficient of weighted network $\mathcal{C}_{w}$, (b) average path length of weighted network $\mathcal{L}_{w}$.

The coupled maps are identical. In the fully developed chaotic regime they are not synchronized, i.e., the evolution of the (chaotic) maps due to their distinct initial conditions is different. Varying the coupling constant $\varepsilon$ changes the dynamical regime of the maps. According to the high chaos in the FDT, each RN has a rather different topology compared to the others, i.e., all the single layers of the MRN are quite different. The BMD is a less chaotic regime, thus, the single layers of the MRN are less different than in the FDT regime (increasing the interlayer similarity). The PPS and STI regimes are closer to each other as both regimes exhibit periodic dynamics but at different scales. Therefore, distinguishing the transition between PPS and STI is the hardest task. Nevertheless, $I$ and $\omega$ are able to detect the corresponding regime transitions. However, $\omega$ performs better, because it is a spatiotemporal measure, i.e., it checks the existence of edges at the same locations in the different layers where the calculation for $I$ does not take the nodes' identities into account.

\section{REAL-WORLD EXAMPLE: MULTIVARIATE PALAEOCLIMATE ANALYSIS}

So far we have been testing the performance of the new MRN method using prototypical models. As a real-world application with changing dynamics and with several variables, we treat a palaeoclimate problem. The investigation of the linkage between the climate conditions and specific environmental responses as well as of changes in these relationships represent an important scientific challenge in palaeoclimatology and ecology in order to improve the understanding of climate impacts and feedback mechanisms. Information on past vegetation is useful to understand the palaeoecological processes linking climate and biosphere changes. Vegetation 


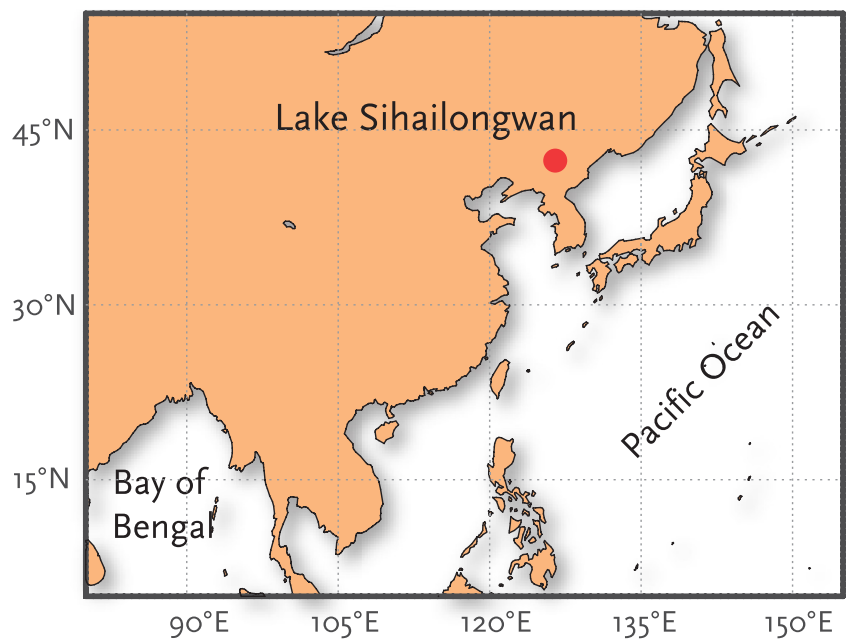

FIG. 6. Location of Lake Sihailongwan from where the pollen record for this study was sampled.

dynamics depends strongly on the environmental conditions and changes in the vegetation dynamics can, therefore, indicate critical changes in the environment (anthropogenic or climate impact).

Pollen assemblages collected from lake sediments are commonly used proxies for palaeoecological and palaeoclimate investigations. Here we focus on the precisely dated late Pleistocene-Holocene pollen record [last 16500 years (16.5 kyr BP)] from the Sihailongwan Lake $\left(42^{\circ} 170^{\prime} \mathrm{N}, 126^{\circ} 360^{\prime} \mathrm{E}\right)$, located in the Longgang volcanic field, Jilin Province, NE China (Fig. 6) [56-58].

Sihailongwan Lake is situated near the northern edge of the Asian summer monsoon system. Originally the Sihailongwan pollen record consists of 103 different pollen taxa, whereby several of them contain redundant information. We, therefore, selected seven tree pollen taxa (Pinus koraiensis, Betula, Carpinus, Juglans, Quercus, Ulmus, Fraxinus, and one herbaceous genus (Artemisia), representing typical regional vegetation elements with different sensitivities on specific environmental conditions during the past $16.5 \mathrm{kyr}$ BP (Fig. 7).

Since the pollen record is irregularly sampled, we first interpolate the data into $N=3500$ points leading to the time resolution $\Delta t=t_{i+1}-t_{i} \approx 4.95 \mathrm{yr} \forall i \in[0, N-1]$. In order to analyze the temporal variation in the environmental dynamics, we apply a sliding window approach consisting of 150 data points per window. By this choice, each window covers about $740 \mathrm{yr}$ and is suitable to represent regime changes in the environmental dynamics. A MRN is created for each window one by one as the window slides over the time series with $90 \%$ overlap.

The application of the MRN technique to the multivariate pollen data set reveals distinct fluctuations of $\omega$ (Fig. 7). A bootstrapping statistical test is applied to evaluate these variations with respect to the null hypothesis that multivariate dynamics is constant over time within $95 \%$ confidence [59].

The considered measure $\omega$ quantifies the amount of similar dynamics in the different pollen taxa. The higher $\omega$, the more similar vary the different vegetation types in the sense of recurrence properties. This means that it is not necessary that

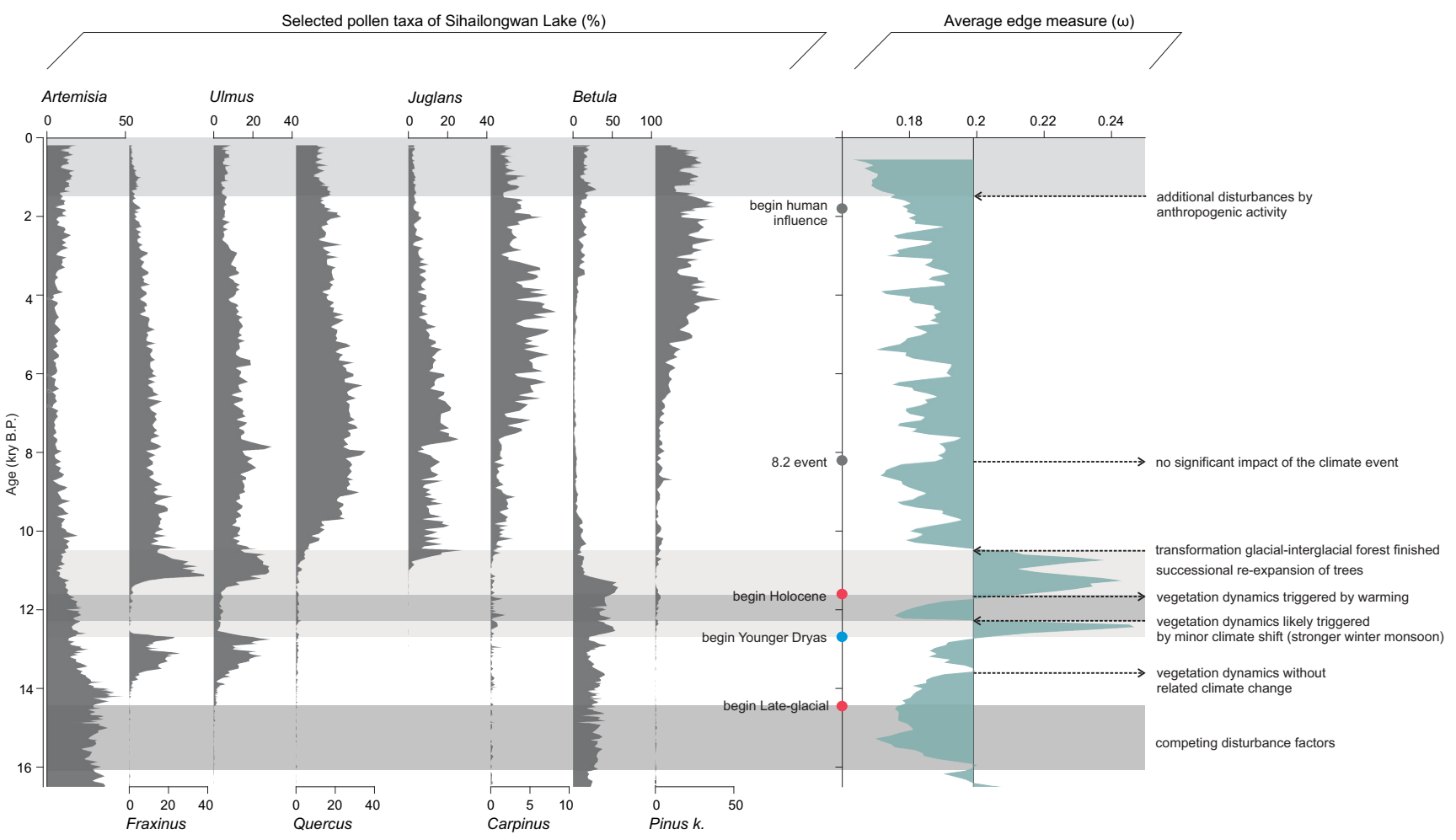

FIG. 7. Pollen data set from Sihalongwan Lake (left) and derived average edge measure $\omega$ calculated in moving windows (right). The base value of the shading (0.199) marks the upper $95 \%$ confidence interval, i.e., exceeding this value corresponds with highly significant increase of $\omega$. The grey shaded bands mark important environmental and climate transitions (see text). 
the pollen abundances go into the same directions, but that, e.g., periodical variations are similar. Low $\omega$ values indicate, in contrast, less similar behavior. This could happen, e.g., when the system is disturbed and some vegetation populations recover faster than others.

The pollen assemblages reveal relatively open, colddry adapted vegetation at Sihailongwan at the end of the pleniglacial. Starting at 16.0 kyr BP, we find a decrease of $\omega$ until $15.0 \mathrm{kyr}$ BP. The apparent decrease of the similarity in the floristic response likely results from rather instable environmental conditions or competing factors influencing the vegetation dynamics. The instable environment at Sihailongwan is strongly evidenced by the sediment composition during this time interval. In particular, the occurrence of graded event layers with reworked soil material implies prevailing permafrost conditions with reduced seepage and erosion events during the final stage of the last glacial period [56]. Moreover, the youngest Heinrich event (H1; 16.0 and $14.6 \mathrm{kyr} \mathrm{BP})$, may have resulted in stronger winter monsoon [60]. By contrast, the ongoing change of $\mathrm{CO}_{2}$ from the glacial to the interglacial level might have individually affected both the physiology of the prevailing plants and the vegetation dynamics in an increasingly less moisture-stressed environment [61,62].

Subsequent to the $\mathrm{H} 1$ event, the environmental conditions during the late-glacial interstadial changed to warmer and moister climate, obviously related with a general change in the regional vegetation to one of more similar vegetation dynamics. The short positive excursion of $\omega$ at $13.6 \mathrm{kyr}$ BP is, however, clearly not related to a climate shift, but most likely results from a change in interspecific competition after reaching a critical population density of Fraxinus [56].

The Younger Dryas temperature decline about 12.7 kyr BP ago is one of the most prominent climate changes observed in Northern Hemisphere palaeoclimate records. Noticeable changes in the Sihailongwan pollen record provide a clear indication of a Younger-Dryas-like cooling in NE China beginning at $12.7 \mathrm{kyr}$ BP. Unlike the abrupt termination of the Younger Dryas in the North Atlantic evidence, the more gradual changes of pollen assemblages indicate a successive substitution of the prevailing boreal woodland by species-rich broadleaf deciduous forests at the Sihailongwan lake during the first millennium of the Holocene (11.7 and 10.7 kyr BP).

Our MRN analysis reveals significant high values of $\omega$ in the period between 12.7 and $10.5 \mathrm{kyr} \mathrm{BP}$, which are interrupted by an abrupt drop in $\omega$ between about 12.3 and $11.7 \mathrm{kyr}$ BP. The timing of this prominent change in vegetation dynamics corresponds to the recently detected bipartition of the Younger Dryas at Suigetsu Lake (Japan) [63], but neither the composition of pollen assemblages nor the sediments of the Sihailongwan sequence reveal a substantial environmental shift at $12.3 \mathrm{kyr}$ BP. It is likely that the ecosystem at Sihailongwan Lake may not be as sensitive to changes in winter conditions as the Japanese site [63], so that the climate shift may have influenced the intrinsic vegetation dynamics at Sihailongwan, but could not trigger substantial floristic and/or landcover changes.

At approximately $10.7 \mathrm{kyr} \mathrm{BP}$, the dynamic spread of thermophilous Juglans and a more gradual increase of Quercus mark a shift in the vegetation composition to oak- and walnutrich mixed conifer hardwood forests. Subsequent to this transition, the MRN analysis data indicate persistent vegetation dynamics, but the general shift to lower $\omega$ values implies less similar behavior of the pollen taxa. Interestingly, a shift in $\omega$ can be observed around $8.2 \mathrm{kyr} \mathrm{BP}$, which might be related to the most prominent drop of the global temperatures during the Holocene. However, as its amplitude does not exceed that of other Holocene changes in vegetation dynamics, the shift does not prove a significant impact of the 8.2 event on the vegetation in NE China.

While substantial human impact cannot be traced by the pollen assemblages, monsoon changes, forest succession dynamics, and flowering activity may therefore primarily drive the observed dynamics in the pollen values or vegetation at Sihailongwan throughout the Holocene. Nevertheless, weak indication of farming activity in conjunction with fires and modest grazing may explain the minor shift to lower values from $1.8 \mathrm{yr}$ BP.

Taken together, in our example we find that the patterns of vegetation dynamics are related to both intrinsic vegetation dynamics and external disturbance factors like climate changes, erosion, or human impact. Obviously, the different vegetation types behave more similarly when one environmental factor acts as the dominant driving force. This is particularly evident at the change to cooler and drier conditions at the beginning of Younger Dryas. Also the rapid mass expansion or the collapse of one or more tree species can have a similar effect on the dynamics of vegetation, as detected in our example at 13.6 kyr BP and throughout the first millennium of the Holocene. On the other hand, less similar behavior of the vegetation results from weaker or competing disturbance factors. Our example demonstrates that the MRN technique goes beyond the classical interpretation of the pollen amplitude variation as a proxy of environmental conditions. A better understanding of this part of the climate-biosphere interaction is of crucial importance as nonlinear feedback mechanisms and tipping points cause high uncertainty and an unpredictable future for humankind $[64,65]$.

\section{CONCLUSIONS}

In this study we have introduced a multiplex recurrence network (MRN) approach which allows us to analyze $m$ dimensional multivariate data simultaneously via joining $m$ recurrence networks (RNs) together. Analyzing and understanding the characteristics of single or low dimensional data with RNs is a known approach [7,37]. Nevertheless, RN analysis of high dimensional systems is not trivial, because the increasing dimension of the phase space would require longer time series. However, studies of real-world problems are often linked with short time series and long time series are often not available. Our approach considers each observation variable in its own phase space and combines them later on. Therefore, it is possible to analyze relatively short multivariate time series with MRNs.

Our extensive tests of the method have demonstrated that the MRN approach enables us to detect abrupt regime transitions accurately in large dimensional systems. It can be used to understand the underlying dynamical properties of prototypical models and real-world applications.

By analyzing a pollen data set for the last $16 \mathrm{kyr}$, we are able to identify periods when different vegetation types 
behave more similarly and periods of less similar behavior. The changes in the vegetation dynamics coincide well with known climate transitions and the increasing human impact. This analysis goes beyond the classical interpretation of the pollen amplitude variation as a proxy of environmental conditions. This view can provide insights into plant communities and their dynamics with respect to climate responses.

Especially for some research fields such as palaeoclimate (pollen taxa, planktonic samples, etc.), neuroscience (short time crises in EEG), economics (stock markets, currencies), where many dependent signals are collected at the same time, our method provides a quantitative and objective way to investigate their dynamics and detects hidden regime changes.

\section{ACKNOWLEDGMENTS}

This work has been supported by German-Israeli Foundation for Scientific Research and Development (GIF), GIF Grant No. I-1298-415.13/2015, and the European Union's Horizon 2020 Research and Innovation program under the Marie Skłodowska-Curie Grant Agreement No. 691037 (QUEST).
[1] H. Poincaré, Acta Math. 13, 1 (1890).

[2] A. Katok and B. Hasselblatt, Introduction to the Modern Theory of Dynamical Systems (Cambridge University Press, Cambridge, England, 1995).

[3] J.-P. Eckmann, S. Oliffson Kamphorst, and D. Ruelle, Europhys. Lett. 4, 973 (1987).

[4] N. Marwan, M. C. Romano, M. Thiel, and J. Kurths, Phys. Rep. 438, 237 (2007).

[5] L. L. Trulla, A. Giuliani, J. P. Zbilut, and C. L. Webber Jr., Phys. Lett. A 223, 255 (1996).

[6] N. Marwan, N. Wessel, U. Meyerfeldt, A. Schirdewan, and J. Kurths, Phys. Rev. E 66, 026702 (2002).

[7] J. Donges, R. Donner, M. Trauth, N. Marwan, H. Schellnhuber, and J. Kurths, Proc. Natl. Acad. Sci. U.S.A. 108, 20422 (2011).

[8] B. H. Jansen, Int. J. Bio-Med. Comput. 27, 95 (1991).

[9] D. Kaplan, M. Furman, S. Pincus, S. Ryan, L. Lipsitz, and A. Goldberger, Biophys. J. 59, 945 (1991).

[10] M. A. Riley, R. Balasubramaniam, and M. T. Turvey, Gait \& Posture 9, 65 (1999).

[11] Y. Neuman, N. Marwan, and D. Livshitz, Complexity 15, 28 (2009).

[12] S. Carrubba, A. Minagar, A. L. Chesson Jr., C. Frilot II, and A. A. Marino, Neurol. Res. 34, 286 (2012).

[13] G. R. Richards, Quat. Sci. Rev. 13, 709 (1994).

[14] N. Marwan, M. H. Trauth, M. Vuille, and J. Kurths, Clim. Dyn. 21, 317 (2003).

[15] T. Matcharashvili, T. Chelidze, and J. Peinke, Nonlin. Dyn. 51, 399 (2008).

[16] I. Ozken, D. Eroglu, T. Stemler, N. Marwan, G. B. Bagci, and J. Kurths, Phys. Rev. E 91, 062911 (2015).

[17] D. Eroglu, F. H. McRobie, I. Ozken, T. Stemler, K.-H. Wyrwoll, S. F. M. Breitenbach, N. Marwan, and J. Kurths, Nat. Commun. 7, 12929 (2016).

[18] N. Asghari, C. Broeg, L. Carone, R. Casas-Miranda, J. C. C. Palacio, I. Csillik, R. Dvorak, F. Freistetter, G. Hadjivantsides, H. Hussmann, A. Khramova, M. Khristoforova, I. Khromova, I. Kitiashivilli, S. Kozlowski, T. Laakso, T. Laczkowski, D. Lytvinenko, O. Miloni, R. Morishima, et al., Astron. Astrophys. 426, 353 (2004).

[19] N. V. Zolotova, D. I. Ponyavin, N. Marwan, and J. Kurths, Astron. Astrophys. 503, 197 (2009).

[20] D. Eroglu, T. K. D. M. Peron, N. Marwan, F. A. Rodrigues, L. F. Costa, M. Sebek, and Z. Kiss, Phys. Rev. E 90, 042919 (2014).

[21] N. Marwan, Eur. Phys. J.: Spec. Top. 164, 3 (2008).
[22] P. Grassberger and I. Procaccia, Physica D (Amsterdam) 9, 189 (1983).

[23] P. Grassberger and I. Procaccia, Physica D 13, 34 (1984).

[24] J. Zhang and M. Small, Phys. Rev. Lett. 96, 238701 (2006).

[25] M. Small, Complex networks from time series: Capturing dynamics, in 2013 IEEE International Symposium on Cicuits Systems (ISCAS2013) (IEEE, 2013), pp. 2509-2512.

[26] L. Lacasa, B. Luque, F. Ballesteros, J. Luque, and J. C. Nuño, Proc. Natl. Acad. Sci. U.S.A. 105, 4972 (2008).

[27] L. Lacasa, B. Luque, J. Luque, and J. C. Nuño, Europhys. Lett. 86, 30001 (2009).

[28] L. Lacasa, V. Nicosia, and V. Latora, Sci. Rep. 5, 15508 (2015).

[29] J. Zhang, J. Sun, X. Luo, K. Zhang, T. Nakamura, and M. Small, Physica D (Amsterdam) 237, 2856 (2008).

[30] X. Xu, J. Zhang, and M. Small, Proc. Natl. Acad. Sci. U.S.A. 105, 19601 (2008).

[31] N. Marwan, J. F. Donges, Y. Zou, R. V. Donner, and J. Kurths, Phys. Lett. A 373, 4246 (2009).

[32] R. V. Donner, M. Small, J. F. Donges, N. Marwan, Y. Zou, R. Xiang, and J. Kurths, Int. J. Bifurcation Chaos 21, 1019 (2011).

[33] P. Grassberger and I. Procaccia, Phys. Rev. Lett. 50, 346 (1983).

[34] V. Afraimovich, Chaos 7, 12 (1997).

[35] B. Saussol, S. Troubetzkoy, and S. Vaienti, J. Stat. Phys. 106, 623 (2002).

[36] B. Saussol and J. Wu, Nonlinearity 16, 1991 (2003).

[37] R. V. Donner, J. Heitzig, J. F. Donges, Y. Zou, N. Marwan, and J. Kurths, Eur. Phys. J. B 84, 653 (2011).

[38] O. Afsar, D. Eroglu, N. Marwan, and J. Kurths, Europhys. Lett. 112, 10005 (2015).

[39] G. M. Ramírez Ávila, A. Gapelyuk, N. Marwan, T. Walther, H. Stepan, J. Kurths, and N. Wessel, Philos. Trans. R. Soc. London A 371, 20110623 (2013).

[40] J. H. Feldhoff, R. V. Donner, J. F. Donges, N. Marwan, and J. Kurths, Phys. Lett. A 376, 3504 (2012).

[41] G. Bianconi, Phys. Rev. E 87, 062806 (2013).

[42] V. Nicosia, G. Bianconi, V. Latora, and M. Barthelemy, Phys. Rev. Lett. 111, 058701 (2013).

[43] M. De Domenico, A. Solé-Ribalta, E. Cozzo, M. Kivelä, Y. Moreno, M. A. Porter, S. Gómez, and A. Arenas, Phys. Rev. X 3, 041022 (2013).

[44] M. Kivelä, A. Arenas, M. Barthelemy, J. P. Gleeson, Y. Moreno, and M. a. Porter, J. Complex Networks 2, 203 (2014).

[45] V. Nicosia and V. Latora, Phys. Rev. E 92, 032805 (2015).

[46] N. H. Packard, J. P. Crutchfield, J. D. Farmer, and R. S. Shaw, Phys. Rev. Lett. 45, 712 (1980). 
[47] H. Kantz and T. Schreiber, Nonlinear Time Series Analysis (Cambridge University Press, Cambridge, England, 1997).

[48] D. Eroglu, N. Marwan, S. Prasad, and J. Kurths, Nonlinear Processes Geophys. 21, 1085 (2014).

[49] C. Ahlstrom, P. Hult, and P. Ask, Thresholding distance plots using true recurrence points, in Proceedings of the IEEE Conference on Acoustics, Speech and Signal Processing (ICASSP 2006) (IEEE, 2006), pp. III688-III691.

[50] S. Schinkel, O. Dimigen, and N. Marwan, Eur. Phys. J.: Spec. Top. 164, 45 (2008).

[51] A. Barrat, M. Barthélemy, R. Pastor-Satorras, and A. Vespignani, Proc. Natl. Acad. Sci. U.S.A. 101, 3747 (2004).

[52] S. Boccaletti, V. Latora, Y. Moreno, M. Chavez, and D. U. Hwang, Phys. Rep. 424, 175 (2006).

[53] K. Kaneko, Chaos 2, 279 (1992).

[54] K. Kaneko, Physica D (Amsterdam) 34, 1 (1989).

[55] K. Kaneko and I. Tsuda, Complex Systems: Chaos and Beyond A Constructive Approach with Applications in Life Sciences (Springer-Verlag, Berlin, Heidelberg, 2001).

[56] M. Stebich, J. Mingram, J. Han, and J. Liu, Glob. Planet. Change 65, 56 (2009).

[57] M. Stebich, K. Rehfeld, F. Schlütz, P. E. Tarasov, J. Liu, and J. Mingram, Quat. Sci. Rev. 124, 275 (2015).
[58] M. Stebich, K. Rehfeld, F. Schlütz, P. E. Tarasov, J. Liu, and J. Mingram, PANGAEA (2015), doi:10.1594/PANGAEA.852704.

[59] N. Marwan, S. Schinkel, and J. Kurths, Europhys. Lett. 101, 20007 (2013).

[60] Y. J. Wang, Science 294, 2345 (2001).

[61] S. A. Cowling and M. T. Sykes, Quat. Res. 52, 237 (1999).

[62] E. Monnin, E. J. Steig, U. Siegenthaler, K. Kawamura, J. Schwander, B. Stauffer, T. F. Stocker, D. L. Morse, J.-M. Barnola, B. Bellier, D. Raynaud, and H. Fischer, Earth Planet. Sci. Lett. 224, 45 (2004).

[63] G. Schlolaut, A. Brauer, T. Nakagawa, H. F. Lamb, J. J. Tyler, R. A. Staff, M. H. Marshall, C. Bronk Ramsey, C. L. Bryant, and P. E. Tarasov, Sci. Rep. 7, 44983 (2017).

[64] T. M. Lenton, H. Held, E. Kriegler, J. W. Hall, W. Lucht, S. Rahmstorf, and H. J. Schellnhuber, Proc. Natl. Acad. Sci. U.S.A. 105, 1786 (2008).

[65] J. Rockstrom, W. Steffen, K. Noone, A. Persson, F. S. Chapin III, E. F. Lambin, T. M. Lenton, M. Scheffer, C. Folke, H. J. Schellnhuber, B. Nykvist, C. A. de Wit, T. Hughes, S. van der Leeuw, H. Rodhe, S. Sorlin, P. K. Snyder, R. Costanza, U. Svedin, M. Falkenmark, et al., Nature (London) 461, 472 (2009). 